\title{
Influence of horse breed on transepidermal water loss
}

\author{
M.P. Szczepanik ${ }^{1}$, P.M. Wilkołek ${ }^{1}$, L.R. Adamek ${ }^{1}$, M. Pluta ${ }^{2}$, M. Gołyńskii ${ }^{3}$, \\ W. Sitkowski ${ }^{1}$, G. Kalisz ${ }^{1}$, I. Taszkun ${ }^{1}$, Z.J.H Pomorski ${ }^{1}$ \\ ${ }^{1}$ Subdepartment of Clinical Diagnostics and Veterinary Dermatology, Faculty of Veterinary Medicine, \\ University of Life Sciences in Lublin, Głęboka 30, 20-612 Lublin, Poland \\ ${ }^{2}$ Equine Breeding and Management Unit, Department of Biology and Animal Breeding, \\ University of Life Sciences in Lublin, Akademicka 13, 20-950 Poland \\ ${ }^{3}$ Subdepartment of Internal Diseases of Farm Animals and Horses, University of Life Sciences in Lublin, \\ Głęboka 30, 20-612 Lublin, Poland
}

\begin{abstract}
Non-invasive methods of skin condition assessment include, among others, the evaluation of transepidermal water loss (TEWL). The aim of the study was to examine whether TEWL values depend on horse breed. The study was conducted on four breeds: "Felin" ponies (FP) $(n=16)$, Polish koniks $(\mathrm{PK})(\mathrm{n}=15)$, Polish cold-blooded horses $(\mathrm{PcbH})(\mathrm{n}=11)$ and Wielkopolska horses $(\mathrm{WH})$ $(n=12)$. It was found that horse breed influences TEWL values. In the neck region, statistically significant differences were found between PK and FP $(\mathrm{p}=0.006)$, and PK and WH $(\mathrm{p}=0.0005)$. In the lumbar region, there were statistically significant differences between FP and PK $(p=0.0009)$, FP and PcbH $(\mathrm{p}=0.0016)$ as well as between PK and WH $(\mathrm{p}=0.000037)$, and $\mathrm{PcbH}$ and WH $(\mathrm{p}=0.0006)$. In the inguinal region statistically significant differences were found between FP and PK $(p=0.0003)$, FP and PcbH $(p=0.0005)$, PK and WH $(p=0.009)$ and PcbH and WH $(p=0.006)$. In the lip region statistically significant differences were observed between FP and PK $(p=0.013)$ as well as between PK and PcbH $(p=0.029)$ and PK and WH $(p=0.009)$. In the examination of TEWL animal breed should be taken into consideration. The non-significant differences found in three of the examined body regions may suggest that these regions are the most adequate for TEWL assessment.
\end{abstract}

Key words: horses, skin, transepidermal water loss

\section{Introduction}

Non-invasive methods used commonly in the process of assessing skin condition include the examination of a number of biophysical skin parameters such as transepidermal water loss (TEWL), skin hydration (SH) or skin $\mathrm{pH}$ and erythema intensity (Beco et al.
2000, Hestler et al. 2004, Shimada et al. 2009, Szczepanik et al. 2011, 2012, 2013). The methods have been widely applied in human medicine to assess skin condition in such diseases as atopic dermatitis or contact dermatitis as well as to monitor the efficiency of locally applied treatments (Korte 1995, Biro et al. 2003, Choi et al. 2003, Grupta et al. 2008, Aschoff 
et al. 2009). The influence of body region on TEWL values has been widely investigated in humans (Marrakchi and Maibach 2007). The methods in question are also becoming increasingly popular in veterinary medicine, especially in scientific research. Biophysical skin parameters (TEWL, SH, skin $\mathrm{pH}$ ) have been assessed in dogs, cats, horses and laboratory animals (Matousek and Campbell 2002, Watosn et al. 2002, Popiel and Nicpoń 2004, Fluhr et al. 2006, Yoshihara et al. 2007, Shimada et al. 2008, Szczepanik et al. 2011, 2012, 2013, Gołyński et al. 2014). The most commonly applied parameter has been TEWL, and most of the studies have concentrated on dogs. This parameter has been assessed in both clinically healthy animals and animals with atopic dermatitis (Beco and Fontaine 2000, Zając et al. 2014, 2015a,b). It has also been used to monitor the efficiency of locally applied medicines (Marsella et al 2006, Shimada et al. 2009, Hightower et al. 2010, Cornegliani et al. 2011, Eui-Hwa Nam et al. 2012). Correlations have been reported between TEWL values and clinical condition of the skin in some of body regions examined (assessment performed with CADESI) (Marsella 2012, Zając 2014). Also, the effects of breed, age and body regions on the results obtained have been investigated (Young et al 2002, Bourdeau et al. 2004, Oh and Oh 2009a,b, Hightower et al. 2010, Szczepanik et al. 2011, 2012, 2013). Measuring TEWL in different body regions (skin mapping) has been performed in dogs, cats and horses and it was found that, similarly to examinations in humans, TEWL values differ according to body region under investigation (Oh and $\mathrm{Oh} 2009 \mathrm{~b}$, Szczepanik et al. 2011, 2012, 2013, Momota et al. 2013). As regards dogs, it has also been shown that breed affects measurement results (Young et al. 2002, Hester et al. 2004). In horses, studies dealing with the measurement of biophysical parameters are scarce. Also, there have been no studies so far investigating the effect of breed on TEWL values. The aim of the present study was to examine whether, and to what extent, horse breed influences TEWL assessment results.

\section{Materials and Methods}

The guidelines of the Animal Care and Ethics Committe of the Univeristy of Life Scinces in Lublin (approval No. 67/2011 and 38/2013) were followed for all animal experiments.

TEWL measurements were performed with four different horse breeds: "Felin" ponies (FP), Polish koniks (PK), Polish cold-blooded horses (PcbH) and Wielkopolska horses (WH). The Felin ponies group comprised 16 animals aged 2-23 years (median age 12 years), 11 males (including 11 castrated males) and 5 females. The Polish koniks group consisted of 15 animals aged 2-14 years (median age 4 years), 6 males and 9 females. The Polish cold-blooded horses group comprised 11 animals aged 2-16 years (median 5 years), 5 females and 6 males. The Wielkopolska horses group contained 12 animals aged 4-30 years (median 6 years), 4 males (including 1 castrated male) and 8 females. The measurements were performed in stables where the animals had been kept. Only clinically healthy horses with no previous skin diseases were included in the study.

The measurements were performed after the animals had been acclimatized in the test room at least $120 \mathrm{~min}$ prior to the experiment. The temperature in the room was $22+/-4^{\circ} \mathrm{C}$ and the humidity was 56 $+/-10 \%$ (according to Tewameter TM300 instruction manual, relative humidity should range from $30 \%$ to $70 \%$, and temperature from 10 to $30^{\circ} \mathrm{C}$ ). The examination was performed between 2011 and 2014. In all horses the measurements were taken in seven different body regions: the neck, the shoulder, the thorax, the lumbar region, the inguinal region, the lip region and the pinna. Clipping was performed prior to measurement and the size of the clipping site corresponded to the size of the probe $\left(1-2 \mathrm{~cm}^{2}\right)$. The measurement itself was taken about 2 min after clipping. Subsequently, TEWL was measured in each of the regions. For each region, 20 successful measurements were performed and the mean result was calculated. The measurement began with $30 \mathrm{sec}$ contact of the probe with the skin, and lasted about $30 \mathrm{sec}$. TEWL assessment was performed with Courage Khazaka Multi Probe Adapter 5 and a TEWL probe: Tewameter TM 300 (Courage Khazaka, Cologne, Germany) (results in $\mathrm{g} / \mathrm{m}^{2} \mathrm{~h}$ ).

\section{Statistical analysis}

Normal distribution of the results was verified with the Shapiro-Wilk test. Statistical analysis was conducted by the Manne-Whitney $\mathrm{U}$ test at P-values of $p=0.05$. All analyses were performed using Statistica 10 software (Statsoft, Tulsa, OK, USA).

\section{Results}

The performed measurements showed that there were statistically significant differences in TEWL values in different body regions in the horses of different breeds. In the neck, statistically significant differences were observed between $\mathrm{PK}$ and FP $(\mathrm{p}=0.006)$, and $\mathrm{PK}$ and $\mathrm{WH}(\mathrm{p}=0.0005)$. In the lum- 
Table 1. TEWL (results in $\mathrm{g} / \mathrm{m}^{2} \mathrm{~h}$ ) in different body regions in 4 horse breeds.

\begin{tabular}{lcccccccccc}
\hline & \multicolumn{2}{c}{ FP } & \multicolumn{2}{c}{ PK } & \multicolumn{2}{c}{ PcbH } & WH \\
\cline { 2 - 10 } & mean & SD & mean & SD & mean & SD & mean & SD \\
\hline Neck & $12.16^{\mathrm{a}}$ & 4.8 & $23.29^{\mathrm{ab}}$ & 11.59 & 16.05 & 10.49 & $8.52^{\mathrm{b}}$ & 2.38 \\
\hline Shoulder & 17.47 & 4.68 & 24.09 & 9.37 & 25.62 & 17.32 & 23.15 & 18.67 \\
\hline Thorax & 14.21 & 6.02 & 18.27 & 8.91 & 23.17 & 13.64 & 12.63 & 9.43 \\
\hline Lumbar region & $9.71^{\mathrm{ab}}$ & 4.57 & $19.57^{\mathrm{ac}}$ & 4.17 & $18.03^{\mathrm{b}}$ & 3.86 & $10^{\mathrm{c}}$ & 2.05 \\
\hline Inguinal region & $11.89^{\mathrm{ab}}$ & 6.79 & $25.74^{\mathrm{ac}}$ & 9.95 & $28.8^{\mathrm{bd}}$ & 11.88 & $14.25^{\mathrm{cd}}$ & 8.27 \\
\hline Lip region & $22.36^{\mathrm{a}}$ & 11.39 & $33.65^{\mathrm{abc}}$ & 8.73 & $25.4^{\mathrm{b}}$ & 8.37 & $22.27^{\mathrm{c}}$ & 10.48 \\
\hline Pinna & 9.97 & 6.83 & 11.17 & 7.10 & 11.36 & 6.53 & 9.15 & 1.09 \\
\hline
\end{tabular}

a, b, c, d, e - statistically significant differences, FP - Feline Ponies, PK - Polish koniks, PcbH - Polish cold-blooded horses, WH - Wielkopolska Horses, SD - Standard Deviation).

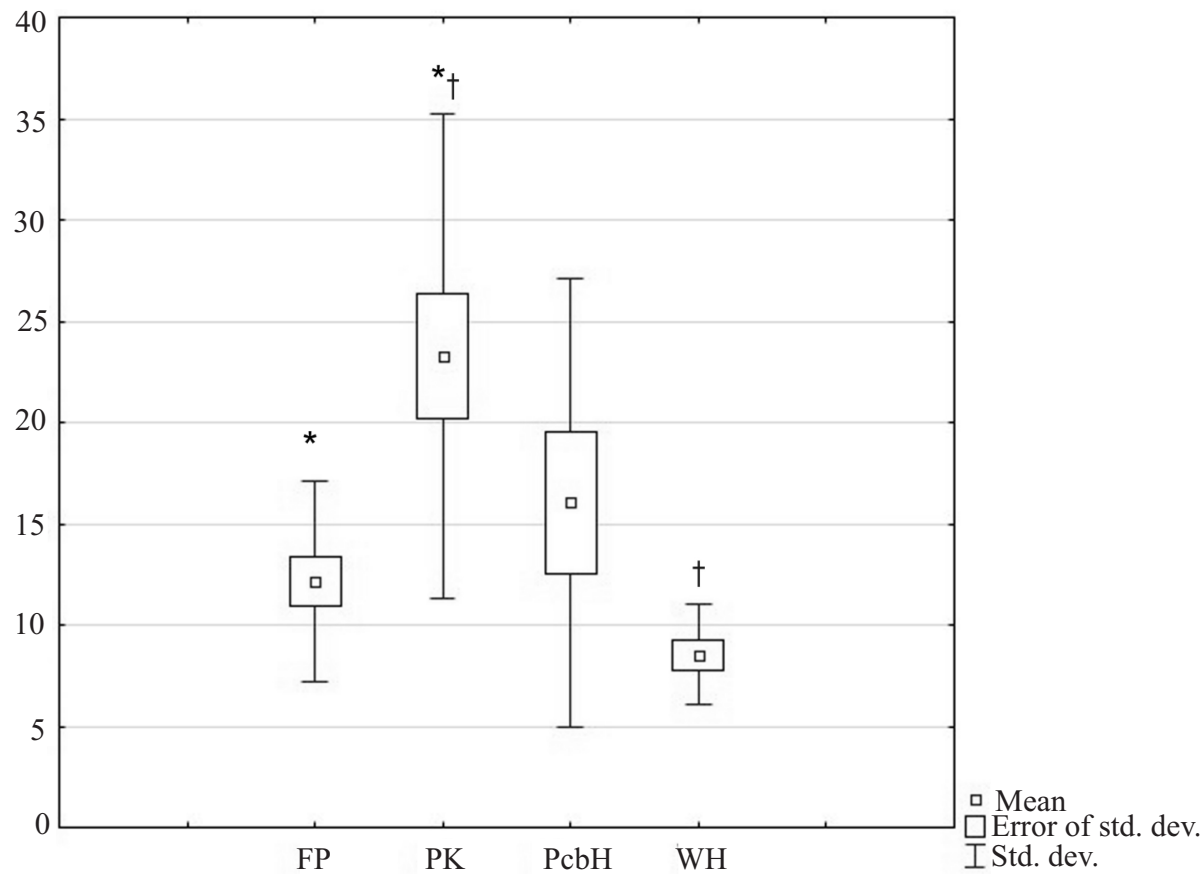

Fig. 1. Mean transepidermal woter loss values in the neck in four horse breeds, results in $\mathrm{g} / \mathrm{m}^{2} \mathrm{~h}(\mathrm{FP}-$ Feline Ponies, PK - Polish koniks, PcbH - Polish cold-blooded horses, WH - Wielkopolska Horses; * $\dagger$ - statistically significant differences, Std. dev. - Standard Deviation).

bar region, there were statistically significant differences between FP and PK ( $p=0.0009)$ and FP and PcbH $(\mathrm{p}=0.0016)$, as well as between $\mathrm{PK}$ and $\mathrm{WH}$ $(p=0.000037)$ and $\mathrm{PcbH}$ and WH $(p=0.0006)$. In the inguinal region, the results differed significantly between FP and PK $(p=0.0003), \quad F P$ and $\mathrm{PcbH}$ $(\mathrm{p}=0.0005), \mathrm{PK}$ and $\mathrm{WH}(\mathrm{p}=0.009)$ and $\mathrm{PcbH}$ and $\mathrm{WH}(\mathrm{p}=0.006)$. In the lip region, statistically significant differences were found between FP and PK $(p=0.013)$ as well as between PK and PcbH $(p=0.029)$ and $\mathrm{PK}$ and $\mathrm{WH}(\mathrm{p}=0.009)$. No statistically significant differences were observed in three body regions, i.e. the pinna, the shoulder and the thorax. The results are shown in Figs. 1-4 (only these regions, where statistically significant differences have been determined).

\section{Discussion}

The present study investigated TEWL values in seven different body regions in four horse breeds. Statistically significant differences between the breeds were reported for the majority of the regions assessed. Most of the differences were observed in the lumbar and inguinal regions (differences present in four cases). Interestingly, no significant differences were 


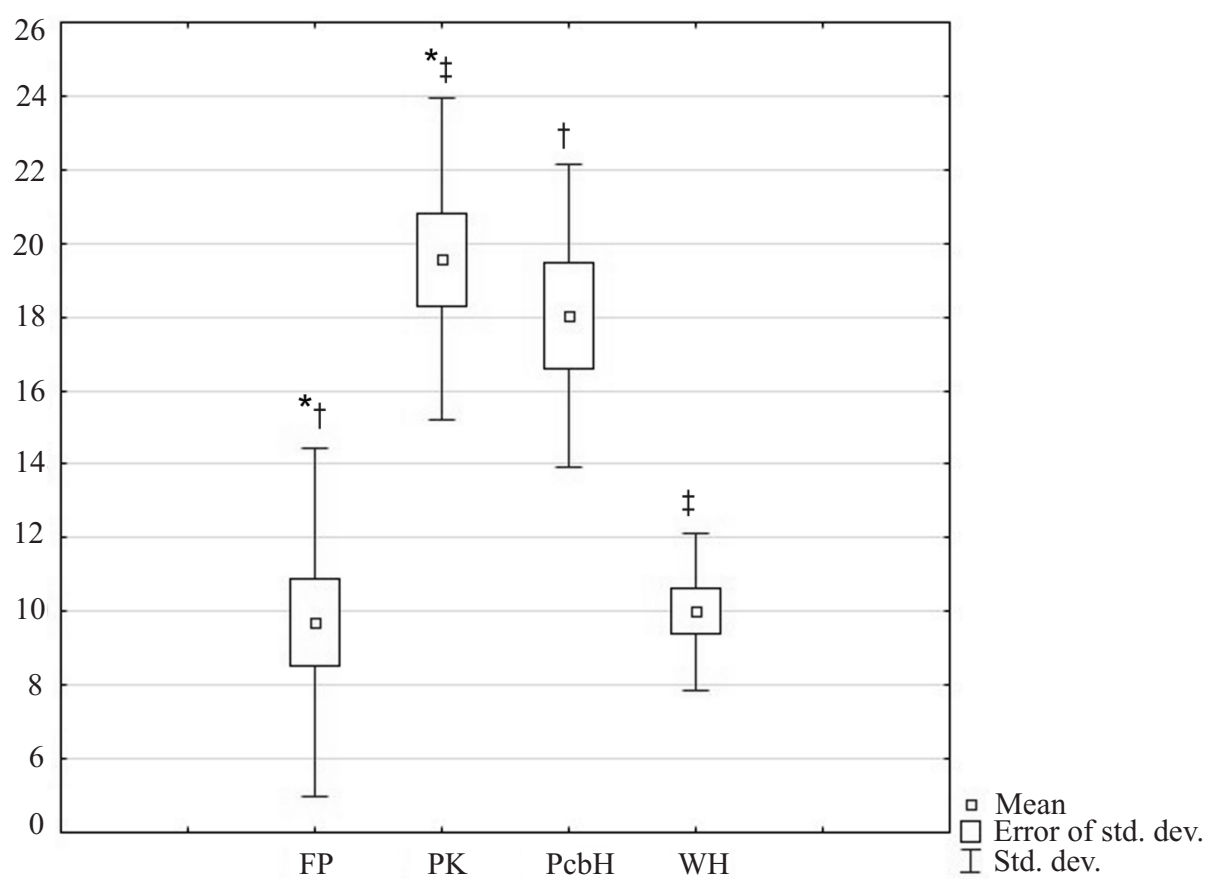

Fig 2. Mean TEWL values in lumbar region in four horse breeds, results in $\mathrm{g} / \mathrm{m}^{2} \mathrm{~h}$ (FP - Feline Ponies, PK - Polish koniks, PcbH - Polish cold-blooded horses, WH - Wielkopolska Horses; * $\uparrow, \ddagger-$ statistically significant differences, Std dev - Standard Deviation).

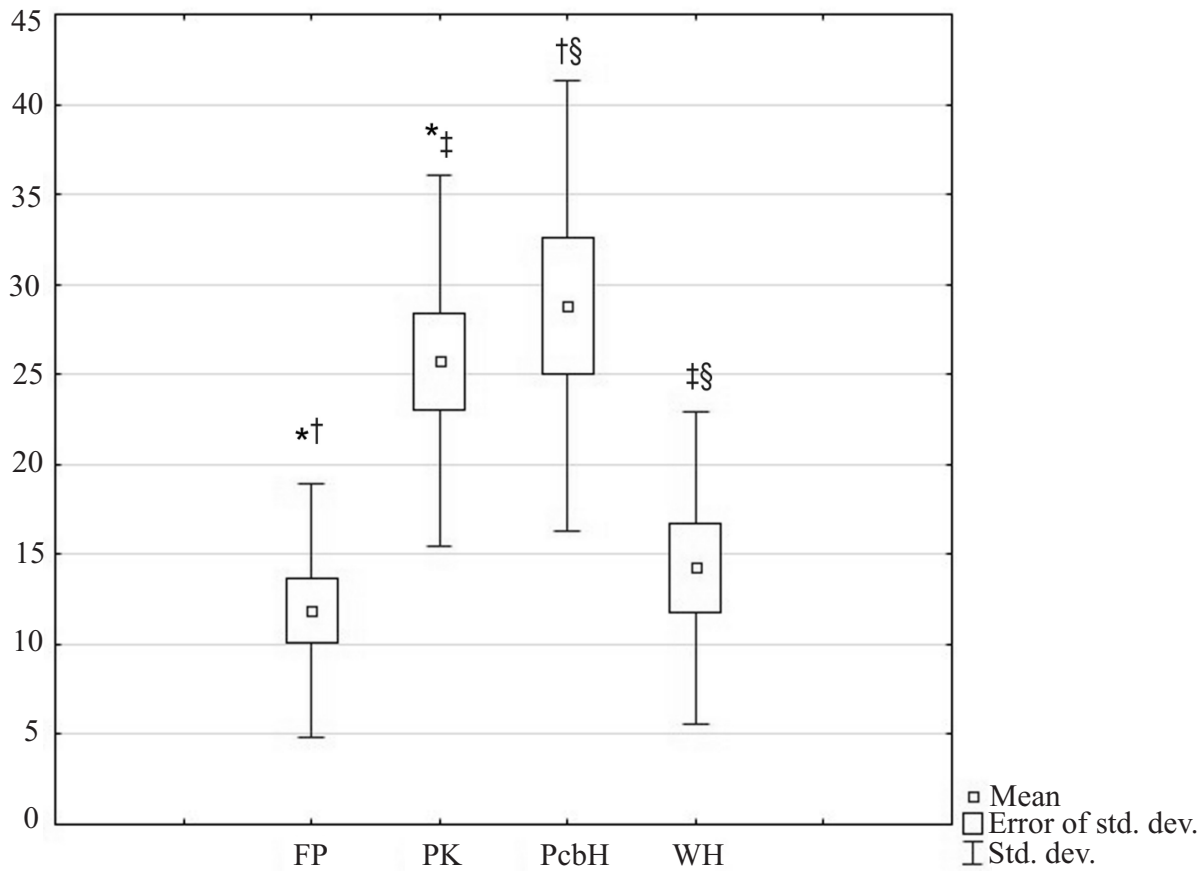

Fig 3. Mean TEWL values in inguinal region in four horse breeds, results in $\mathrm{g} / \mathrm{m}^{2} \mathrm{~h}(\mathrm{FP}-$ Feline Ponies, PK - Polish koniks, PcbH - Polish cold-blooded horses, WH - Wielkopolska Horses; * $\dagger$, $\ddagger$ $\S$ - statistically significant differences, Std. dev. - Standard Deviation).

found between the four breeds in three body regions, i.e. the shoulder, the side of the thorax and the pinna. The comparison of TEWL values in different breeds revealed the most similar results for $\mathrm{FP}$ and $\mathrm{WH}$, where no statistically significant differences were noted in any of the body regions examined. Also, in the case of PK and PcbH the differences were small and only applied to one body region, the lip. The greatest differences were observed between FP and $\mathrm{PK}$ as well as PK and $\mathrm{WH}$, where they were found in four out of seven body regions examined. 


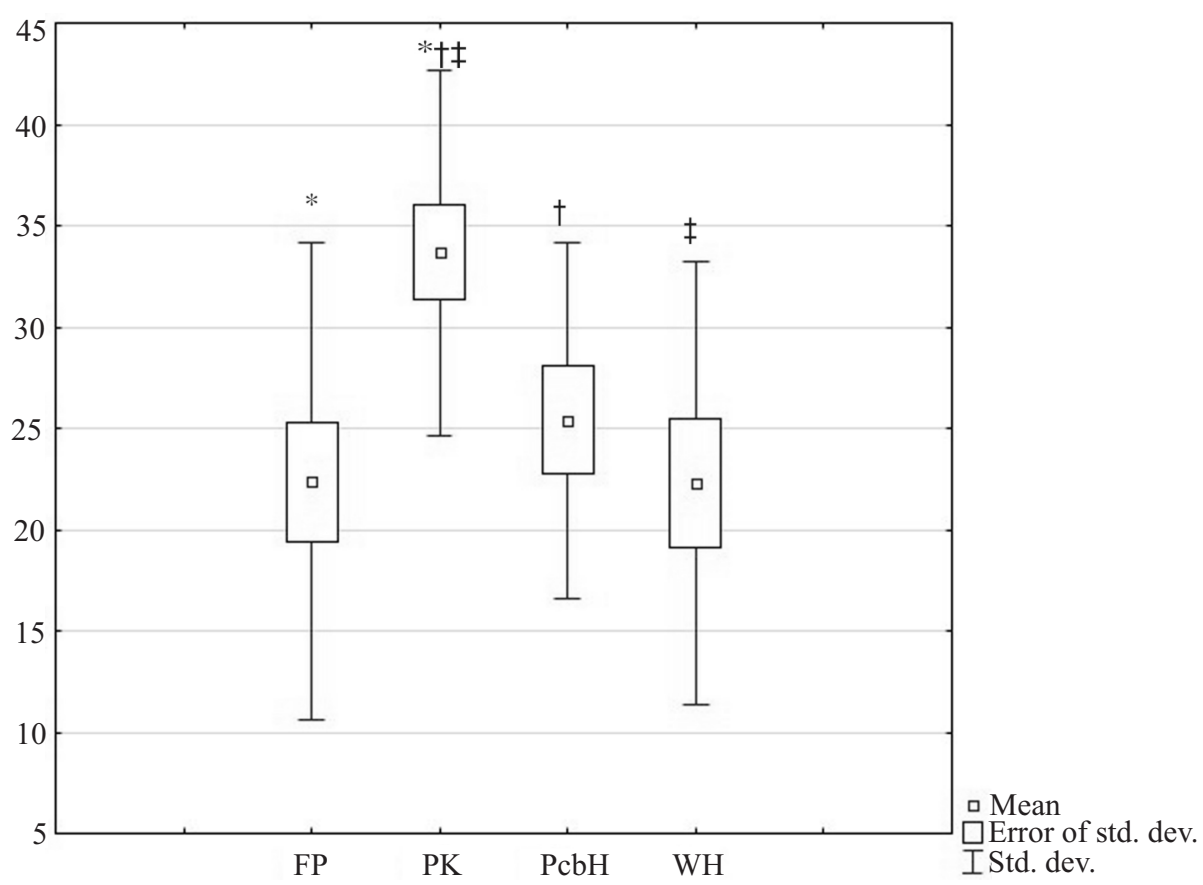

Fig. 4. Mean TEWL values in the lip region in four horse breeds, results in $\mathrm{g} / \mathrm{m}^{2} \mathrm{~h}$ (FP - Feline Ponies, PK - Polish koniks, PcbH - Polish cold-blooded horses, WH - Wielkopolska Horses; *, $†, \ddagger-$ statistically significant differences, Std. dev. - Standard Deviation).

There have been no studies so far dealing with the influence of horse breed on TEWL values. Published research reports on this parameter in horses discussed the effect of sex and body region on TEWL (also skin hydration and skin $\mathrm{pH}$ were examined). These studies corroborated the claim that TEWL values differ significantly according to the body region under investigation (Szczepanik et al. 2012, 2013) and in "Felin" ponies, a greater variability between the body regions assessed was observed (10 statistically significant differences) than in Polish koniks (6 statistically significant differences, only in one body region). Sex of the animals, however, was not found to influence TEWL values. Similarly, body region was found to influence TEWL values in dogs and cats. It was confirmed that the values differ significantly according to body region examined (Oh and Oh 2009b, Szczepanik et al. 2011, Momota et al 2013).

The effect of breed on TEWL values has been assessed in dogs, and it has been demonstrated that it can influence the values of the parameter (Young et al 2002, Hester et al. 2004).

To conclude, the results of the present study show that not only body region but also animal breed should be taken into consideration in the process of TEWL assessment. The lack of statistically significant differences in three out of seven investigated body regions suggests that these regions are the most adequate for examination, due to the fact that the results can be considered comparable irrespective of horse breed. Because of the differences in most of the body regions, we believe, that any scientific research should be made in horses of the same breed. Further investigation are necessary involving different horse breeds and considering measurements in different body regions.

\section{Acknowledgements}

The authors would like to thank the experimental farms in Florianka and Felin (University of Life Sciences in Lublin, Poland).

\section{References}

Aalto-Korte K (1995) Improvement of skin barrier function during treatment of atopic dermatitis. J Am Acad Dermatol 33: 969-972.

Aschoff R, Schwanebeck U, Brautigam M, Meurer M (2009) Skin physiological parameters confirm the therapeutic efficacy of pimecrolimus cream $1 \%$ in patients with mild-to-moderate atopic dermatitis. Exp Dermatol 18: 24-29.

Beco L, Fontaine J (2000) Corneometry and transepidermal water loss measurements in the canine species: validation of these techniques in normal beagle dogs. Ann Med Vet 144: 329-333.

Bourdeau P, Taylor KW, Nguyen P, Biourge V (2004) Evaluation of the influence of sex, diet and time on skin pH and surface lipids of cats. Vet Dermatol 15 (Suppl 1) 41e-69. 
Biro K, Thaçi D, Ochsendorf FR, Kaufmann R, Boehncke WH. (2003) Efficacy of dexpanthenol in skin protection against irritation: a double-blind, placebo-controlled study. Contact Dermatitis 49: 80-84.

Choi SJ, Song MG, Sung WT, Lee DY, Lee JH, Lee ES, Yang JM (2003) Comparison of Transepidermal Water Loss, Capacitance and $\mathrm{pH}$ Values in the Skin between Intrinsic and Extrinsic Atopic Dermatitis Patients. J Korean Med Sci 18: 93-96.

Cornegliani L, Vercelli A, Sala E, Marsella R (2012) Transepidermal water loss in healthy and atopic dogs treated and untreated: a comparitive preliminary study. Vet Dermatol 23: 41-44.

Fluhr JW, Feingold KR, Elias PM (2006) Transepidermal water loss reflects permeability barrier status: validation in human and rodent in vivo and ex vivo models. Exp Dermatol 15: 483-492.

Gołyński M, Szczepanik M, Lutnicki K, Adamek Ł, Gołyńska M, Wilkołek P, Sitkowski W, Kurek Ł, Dębiak $P$ (2014) Biophysical parameters of rats' skin after the administration of methimazole. Bull Vet Inst Pulawy 58: 315-319.

Gupta J, Grube E, Ericksen MB, Stevenson MD, Lucky AW, Sheth AP, Assa'ad AH, Khurana Hershey GK (2008) Intrinsically defective skin barrier function in children with atopic dermatitis correlates with disease severity. J Allergy Clin Immunol 121: 725-730.

Hester SL, Rees CA, Kennis RA, Zoran DL, Bigley KE, Wright AS, Kirby NA, Bauer JE (2004) Evaluation of corneometry (skin hydration) and transepidermal water- loss measurements in two canine breeds. J Nutr 134S: 2110S-2113S.

Hightower K, Marsella R, Flynn-Lurie A (2010) Effects of age and allergen exposure on transepidermal water loss in a house dust mite-sensitized beagle model of atopic dermatitis. Vet Dermatol 21: 88-95.

Marrakchi S, Maibach HI (2007) Biophysical parameters of skin: map of human face, regional, and age-related differences. Contact Derm 57: $28 \mathrm{e} 34$.

Marsella R, Olivry T, Nicklin C, Lopez J (2006) Pilot investigation of a model for canine atopic dermatitis: environmental house dust mite challenge of high-IgE-producing beagles, mite hypersensitive dogs with atopic dermatitis and normal dogs. Vet Dermatol 17: 24-35.

Marsella R (2012) Are transepidermal water loss and clinical signs correlated in canine atopic dermatitis? A compilation of studies. Vet Dermatol 23: 238-e49.

Matousek JL, Campbell KL (2002) A comparative review of cutaneous pH. Vet Dermatol 13: 293-300.

Momota Y, Shimada K, Takami A, Akaogi H, Takasaki M, Mimura K, Azakami D, Ishioka K, Nakamura Y, Sako $\mathrm{T}$ (2013) Transepidermal water loss in cats: comparision of three differently clipped sites to assess the influence of hair coat on transepidermal water loss values. Vet Dermatol 24: 450-452.

Nam EH, Park SH, Jung J, Han SH, Youn HY, Chae JS, Hwang CY (2012) Evaluation of the effect of a $0,0584 \%$ hydrocortisone aceponate spray on clinical signs and skin barrier function in dogs with atopic dermatitis. J Vet Sci 13: $187-191$

Oh WS, Oh TH (2009a) Measurement of transepidermal water loss from clipped and unclipeped anatomical sites on the dog. Aust Vet J 87: 409-412.
Oh WS Oh TH (2009b) Mapping of the dog skin based on biophysical measurements. Vet Dermatol 21: 367-372.

Popiel J, Nicpon J (2004) The correlation of a skin ph of dogs with the pyoderm treatment before and after usage of surface-acting agents of known $\mathrm{pH}$ reaction. Acta Sci Pol Medicina Veterinaria 3: 53-60.

Shimada K, Yoon JS, Yoshihara T, Iwasaki T, Nishifuji K (2009) Increased transepidermal water loss and decreased ceramide content in lesional and non-lesional skin of dogs with atopic dermatitis. Vet Dermatol 20: 541-546.

Shimada K, Yosihara T, Yamamoto M, Konno K, Momoi Y, Nishifuji K, Iwasaki T (2008). Transepidermal water loss (TEWL) reflects skin barrier function of dogs. J Vet Med Sci 70: 841-843.

Szczepanik MP, Wilkołek PM, Adamek ŁR, Pomorski ZJ (2011) The examination of biophysical parameters of skin (transepidermal water loss, skin hydration and $\mathrm{pH}$ value) in different body regions of normal cats of both sexes. J Feline Med Surg 13: 224-230.

Szczepanik MP, Wilkołek PM, Pluta M, Adamek ŁR, Golyński M, Pomorski ZJ, Sitkowski W (2013) The examination of biophysical skin parameters (transepidermal water loss, skin hydration and $\mathrm{pH}$ value) in different body regions in Polish ponies. Pol J Vet Sci 16: 741-747.

Szczepanik MP, Wilkołek PM, Pluta M, Adamek ŁR, Pomorski ZJH (2012) The examination of biophysical parameters of skin (transepidermal water loss, skin hydration and $\mathrm{pH}$ value) in different body regions of ponies. Pol J Vet Science 15: 553-559.

Watson A, Fray T, Clarke S, Yates D, Markwell P (2002) Reliable use of the ServoMed Evaporimeter EP-2 to assess transepidermal water loss in the canine. J Nutr 132S: 1661S-1664S.

Yoshihara T, Shimada K, Momoi Y, Konno K, Iwasaki $\mathrm{T}$ (2007) A new method of measuring the transepidermal water loss (TEWL) of dog skin. J Vet Med Sci 69: 289-292.

Young LA, Dodge JC, Guest KJ, Cline JL, Kerr WW (2002) Age, breed, sex and period effects on skin biophysical parameters for dogs fed canned dog food. J Nutr 132S: 1695S-1697S.

Zając M, Szczepanik MP, Wilkołek PM, Adamek ŁR, Pomorski ZJ, Sitkowski W, Gołynski MG (2014) Assessment of the relationship between transepidermal water loss (TEWL) and severity of clinical signs (CADESI-03) in atopic dogs. Vet Dermatol 25: 503-e83.

Zając M, Szczepanik MP, Wilkołek PM, Adamek ŁR, Pomorski ZJ, Sitkowski W, Gołyński M (2015a) Assessment of a correlation between Canine Atopic Dermatitis Extent and Severity Index (CADESI-03) and selected biophysical skin measures (skin hydration, $\mathrm{pH}$, and erythema intensity) in dogs with naturally occurring atopic dermatitis. Can J Vet Res 79: 136-40.

Zając M, Szczepanik MP, Wilkołek PM, Adamek ŁR, Pomorski ZJ (2015b) The influence of non-specific anti-pruritus treatment with cyclosporine A on transepidermal water loss (TEWL) in natural atopic dermatitis in dogs. Pol J Vet Sci 18: 415-424. 